\title{
Inflation driven by causal heat flux
}

\author{
R. Maartens $\ddagger$, M. Govender $\ddagger$ and S.D. Maharaj $\ddagger$ \\ †School of Computer Science and Mathematics, Portsmouth University, Portsmouth PO1 2EG, England \\ †Department of Mathematics and Applied Mathematics, University of Natal, Durban 4041, South Africa
}

October 1997

\begin{abstract}
We find a simple inflationary solution in an inhomogeneous spacetime with heat flux. The heat flux obeys a causal transport equation, and counteracts the inflationary decrease of energy density. At late times, the heat flux tends to zero and the fluid approaches the equation of state $p=-\rho$.
\end{abstract}

Keywords: cosmology - inflation - nonequilibrium thermodynamics

\section{Introduction}

Inflationary expansion arises when the effective pressure becomes sufficiently negative that its 'repulsive' contribution to gravity becomes dominant. This scenario occurs naturally in scalar-field models of the early universe. On the other hand, various papers have considered the possibility that inflation could occur in an 'ordinary' fluid if there is sufficient bulk viscous stress to drive the effective pressure negative (see, e.g., [1, 2, 3] and references given there). Clearly, as pointed out in 叫, the bulk viscous stress must exceed the local equilibrium pressure, so that such models are far from equilibrium.

Bulk viscous stress is a scalar dissipative effect, and thus compatible with the spatial homogeneity and isotropy of Friedmann-Lemaitre-Roberston-Walker (FLRW) spacetime. The vector dissipative effect of heat flux is not compatible with FLRW symmetry. Here we present a 'toy' model to show that, in a simple inhomogeneous geometry, it is in principle possible for inflationary expansion to arise in a fluid with heat flux. Essentially, the heat flux acts to counter the decrease of energy density $\rho$ due to expansion, while the pressure 
$p$ is steadily reduced (with asymptotic limit $p \rightarrow-\rho$ ). Unlike the viscous inflationary models, the fluid is not far from equilibrium at all spacetime events.

For consistency, we use the causal transport equation of Israel and Stewart for heat flux, in preference to the often used Fourier equation (generalized to relativity), which is noncausal and has unstable equilibrium states [5].

\section{The basic equations}

Consider Modak's shear-free spherically symmetric model, given in comoving coordinates by [6]

$$
d s^{2}=-\left[1+M(t) r^{2}\right]^{2} d t^{2}+a^{2}(t)\left[d r^{2}+r^{2}\left(d \theta^{2}+\sin ^{2} \theta d \phi^{2}\right)\right],
$$

where the fluid four-velocity is $u^{\alpha}=\left[1+M r^{2}\right]^{-1} \delta^{\alpha}{ }_{0}$. The fluid four-acceleration is

$$
a_{\alpha} \equiv u^{\beta} \nabla_{\beta} u_{\alpha}=\left(\frac{2 M r}{1+M r^{2}}\right) \delta_{\alpha}^{1}
$$

and the volume expansion rate is

$$
\Theta \equiv \nabla^{\alpha} u_{\alpha}=\frac{3 H}{1+M r^{2}}, \quad H \equiv \frac{\dot{a}}{a},
$$

where a dot denotes $\partial / \partial t$. The heat flux vector is

$$
q^{\alpha}=\left(\frac{q}{a}\right) \delta_{1}^{\alpha} \quad \text { where } \quad q^{2}(t, r)=q^{\alpha} q_{\alpha}
$$

Note that $q$ is a covariant scalar measure of the heat flux magnitude. The four-velocity $u^{\alpha}$ is comoving with the particle frame, in which the total energy flux is the heat flux, since there is no particle flux relative to this frame.

The Einstein field equations

$$
\begin{aligned}
\rho & =\frac{1}{3} \Theta^{2} \\
p & =\frac{1}{\left(1+M r^{2}\right)^{2}}\left[\frac{2}{3} \dot{M} r^{2} \Theta-2 \frac{\ddot{a}}{a}+\frac{4 M\left(1+M r^{2}\right)}{a^{2}}\right]-\frac{1}{3} \rho \\
q & =-\frac{4 M r \Theta}{3\left(1+M r^{2}\right) a}
\end{aligned}
$$

Then the conservation equations

$$
\begin{aligned}
& u^{\alpha} \nabla_{\alpha} \rho+(\rho+p) \Theta+\nabla^{\alpha} q_{\alpha}+a^{\alpha} q_{\alpha}=0 \\
& (\rho+p) a_{\alpha}+h_{\alpha}{ }^{\beta}\left(\nabla_{\beta} p+u^{\gamma} \nabla_{\gamma} q_{\beta}\right)+\frac{4}{3} \Theta q_{\alpha}=0
\end{aligned}
$$

are identically satisfied, where $h_{\alpha \beta}=g_{\alpha \beta}+u_{\alpha} u_{\beta}$ projects orthogonal to $u^{\alpha}$, and $g_{\alpha \beta}$ is the metric. In Modak's model, the heat flux is radially inward if the fluid is expanding, and

\footnotetext{
${ }^{1}$ We use units with $8 \pi G=1=c$
} 
this is reflected in the fact that the energy density at each instant of time is a maximum at the centre of symmetry.

The functions $a(t)$ and $M(t)$ are determined after appropriate thermodynamic equations are imposed. We now require that the heat flux is governed by the causal transport equation of Israel and Stewart [5]

$$
\tau h^{\alpha \beta} u^{\gamma} \nabla_{\gamma} q_{\beta}+q^{\alpha}=-\kappa\left(h^{\alpha \beta} \nabla_{\beta} T+T a^{\alpha}\right),
$$

where $T$ is the local equilibrium temperature, $\kappa(\geq 0)$ is the thermal conductivity and $\tau$ is the relaxational time-scale which gives rise to the causal and stable behaviour of the theory. The noncausal Fourier transport equation has $\tau=0$ and reduces from an evolution equation to an algebraic constraint on the heat flux. Intuitively, one can see that in this case, the heat flux is instantaneously brought to zero when the temperature gradient and acceleration are 'switched off'.

For the line element (11), equation (10) becomes

$$
\tau \dot{q}+\left(1+M r^{2}\right) q=-\frac{\kappa}{a}\left[\left(1+M r^{2}\right) T\right]^{\prime},
$$

where a prime denotes $\partial / \partial r$.

\section{A simple inflationary model}

To close the system of equations, one needs further thermodynamic information about $\tau$, $\kappa, \rho, p$ and $T$. In [6], this is done for a radiation-dominated model where heat transport arises from radiative transfer. Here we follow a different approach, since we are interested in demonstrating the theoretical possibility of inflation driven by causal heat flux. First we suppose that $M=M_{0}$ is a positive constant. Then on each comoving sphere $r=$ constant of fluid particles, inflationary expansion is characterized by $\ddot{a}>0$. A particular case of inflationary expansion is $H=H_{0}$ where $H_{0}$ is a positive constant, and then $a=a_{0} \exp \left(H_{0} t\right)$. Substituting into equations (5)-(可), we find

$$
\begin{aligned}
\rho & =\frac{3 H_{0}^{2}}{\left(1+M_{0} r^{2}\right)^{2}}, \\
p & =\left[\frac{4 M_{0}}{a_{0}^{2}\left(1+M_{0} r^{2}\right)}\right] e^{-2 H_{0} t}-\rho, \\
q & =-\left[\frac{4 M_{0} H_{0} r}{a_{0}\left(1+M_{0} r^{2}\right)^{2}}\right] e^{-H_{0} t} .
\end{aligned}
$$

Note that the magnitude of the heat flux is a maximum at $r=1 / \sqrt{3 M_{0}}$, falling to zero at the centre and as $r \rightarrow \infty$. The causal transport equation (11) becomes

$$
4 M_{0} H_{0} r\left[\left(1+M_{0} r^{2}\right)-H_{0} \tau\right]=\kappa\left(1+M_{0} r^{2}\right)^{2}\left[\left(1+M_{0} r^{2}\right) T\right]^{\prime} .
$$

We have yet to specify $\kappa, \tau$ and equations of state involving the temperature. However, we will avoid the difficult issue of trying to introduce a microscopically motivated model, 
since our primary aim is only to show the possibility of consistent solutions. In this spirit, we will satisfy the causal transport condition (15) by taking

$$
\begin{aligned}
\tau & =\left(1+M_{0} r^{2}\right) H_{0}^{-1}, \\
T & =\frac{U(t)}{1+M_{0} r^{2}}
\end{aligned}
$$

where $U$ is an arbitrary positive function. Equation (16) implies that the relaxation time is the same as the local expansion time, which is not unreasonable. The radially inward heat flux counters the cooling brought about by expansion. However, one might expect that the inflationary cooling will not be balanced or overcome by dissipative heating, and then one would choose $\dot{U}<0$.

For any choice of $U$, it follows from (17) that $T$ decreases radially outward, in apparent contradiction to the radially inward direction of the heat flux. However, in relativity, the inertia of heat energy gives rise to an accelerative contribution to the heat flux. In our model, the latter dominates the temperature gradient. Note that, whatever the (positive) choice of $U$ and $\kappa$, the second law of thermodynamics will be satisfied, since it is built into the causal theory [5].

The model given by equations (12) $-(14),(16)$ and (17) describes inhomogeneous inflation driven by causal heat flux. The inflationary expansion rapidly reduces the pressure, as shown by equation (13), but the heat flux counters the tendency for the energy density to decrease, and equation (12) shows that the energy density is a comoving constant $\left(u^{\alpha} \nabla_{\alpha} \rho=0\right)$. At late times, the heat flux tends to zero and the fluid approaches a de Sitter-like equilibrium with $p=-\rho$. The deviation of the fluid from equilibrium is measured by the covariant dimensionless ratio

$$
\frac{|q|}{\rho}=\left(\frac{4 M_{0}}{3 a_{0} H_{0}}\right) r e^{-H_{0} t}
$$

At late times, this ratio rapidly becomes small. However for any fixed finite time, the ratio grows with radius, reflecting the fact that $|q|$ decays less rapidly than $\rho$. Thus the fluid is only close to equilibrium near the centre and at late times. By contrast, in bulk viscous inflation the fluid is far from equilibrium at all times and positions.

We have shown that in principle it is possible for causal heat flux to drive inflation. This approach could be the basis for a model of inflationary expansion of bubbles in the early universe, driven by causal energy transport processes. Our model is of course highly simplified, and lacks some of the physical properties that would be expected of a more realistic model. For example, there is no mechanism in our simple model for achieving an exit from inflation, and the model inflates for all time. Furthermore, the heat flux does not homogenize the universe, as one might intuitively expect, so that the metric does not tend to the FLRW metric asymptotically. This is a direct consequence of the simple choice of $M(t)$ as a constant. However, in principle it should be possible to overcome these drawbacks with a more sophisticated and complicated model. 


\section{References}

[1] D Pavon, J Bafaluy and D Jou (1991) Class. Quantum Grav. 8, 347

[2] R Maartens (1995) Class. Quantum Grav. 12, 1455

[3] W Zimdahl (1996) Phys. Rev. D 53, 5483

[4] R Maartens and V Mendez (1997) Phys. Rev. D 55, 1937

[5] WA Hiscock and L Lindblom (1983) Ann. Phys. 151, 466

[6] J Triginer and D Pavon (1995) Class. Quantum Grav. 12, 689. 